\title{
Germanica
}

\section{Engel in der Nacht : l'ange dans la prose de jeunesse d'Ilsé Aichinger}

Engel in der Nacht: Die Figur des Engels in Ilse Aichingers Frühprosa

\section{Brigitte Desbrière-Nicolas}

\section{OpenEdition}

1 Journals

Édition électronique

URL : http://journals.openedition.org/germanica/2254

DOI : $10.4000 /$ germanica.2254

ISSN : 2107-0784

Éditeur

Université de Lille

\section{Édition imprimée}

Date de publication : 1 janvier 1999

ISBN : 2-913857-00-0

ISSN : 0984-2632

\section{Référence électronique}

Brigitte Desbrière-Nicolas, «Engel in der Nacht : I'ange dans la prose de jeunesse d'Ilsé Aichinger »,

Germanica [En ligne], 24 | 1999, mis en ligne le 31 janvier 2014, consulté le 06 octobre 2020. URL

http://journals.openedition.org/germanica/2254 ; DOI : https://doi.org/10.4000/germanica.2254

Ce document a été généré automatiquement le 6 octobre 2020.

(C) Tous droits réservés 


\title{
Engel in der Nacht : l'ange dans la prose de jeunesse d'Ilsé Aichinger
}

\author{
Engel in der Nacht: Die Figur des Engels in Ilse Aichingers Frühprosa
}

Brigitte Desbrière-Nicolas

«Der Himmel ist unterwegs, aber ihr haltet ihn

auf.»

Die größere Hoffnung.

1 En 1952, quatre ans après la parution de son premier et unique roman Die größere Hoffnung ${ }^{1}$, Ilse Aichinger publie un recueil de sept textes en prose ${ }^{2}$ qui propose en guise de préface une réflexion intitulée Über das Erzählen in dieser Zeit (1951) :

Form ist nie aus dem Gefühl der Sicherheit entstanden, sondern immer im Angesicht des Endes... Wenn wir es richtig nehmen, können wir, was gegen uns gerichtet scheint, wenden, wir können gerade vom Ende her und auf das Ende hin zu erzählen beginnen, und die Welt geht uns wieder auf. Dann reden wir, wenn wir unter dem Galgen zu reden beginnen, vom Leben selbst ${ }^{3}$.

2 À la fois titre de récit et d'ouvrage, les Paroles dites sous la potence ne constituent pas l'expression originale d'une inspiration littéraire éphémère, elles indiquent le fondement même de l'écriture.

3 L'ombre menaçante de la potence a plané pendant près d'une décennie sur l'existence de la jeune Viennoise. Ilse Aichinger a dix-sept ans lorsque l'Autriche est annexée et elle subit de plein fouet le cortège des mesures discriminatoires introduites par le nouveau régime. D’origine non-juive par son père, mais juive par l'ascendance maternelle, elle doit supporter non seulement la séparation : sa sœur jumelle réussit in extremis, le 4 juillet 1939, à gagner la Grande-Bretagne, mais aussi les difficultés matérielles: Berta Aichinger qui l'élève seule est privée de son emploi de médecin scolaire et l'aïeule qui les héberge de son appartement. La détresse morale s'intensifie encore en 1942 avec la déportation si longtemps redoutée et sans retour de sa grandmère et de ses tantes. Use est classée «Mischling 1. Grades» par les lois de Nuremberg et, étant baptisée, elle protège jusqu'à sa majorité (et grâce à une extrême vigilance jusqu'en 1945) sa mère des sinistres convois à destination de la Pologne. Chaque jour lui 
apporte son lot d'interrogations et d'angoisses d'autant plus justifiées que dans le cadre des débats sur la solution finale (Conférence de Wannsee, 1942) ${ }^{4}$ la question des demijuifs est encore irrésolue.

4 Alors que ces survivants, Allemands et Autrichiens, pourtant soumis sur leur sol natal à d'incessantes discriminations n'ont retenu que tardivement l'attention des historiens ${ }^{5}$, leurs souffrances constituent la substance même du premier roman d'Ilse Aichinger.

5 Die größere Hoffnung met en effet en scène, dans une succession de tableaux qui pour quelques uns furent publiés de façon autonome ${ }^{6}$, une fillette demi-juive, Ellen, dont la mère réussit à embarquer pour les Etats-Unis. Comme Use, elle est certes dispensée du port de l'étoile jaune, mais elle doit renoncer à l'espoir d'obtenir le visa l'autorisant à émigrer. Avec un groupe d'enfants juifs voués à la mort, elle tente de lutter pour rester debout et partir à la recherche du plus grand espoir reposant - quoiqu'il advienne - sur la foi «daß irgendwo alles blau wird». «Hilf mir!», implore-t-elle Saint François Xavier «über das Wasser zu gehen, auch wenn ich hier bleiben muß» $(\mathrm{gH}, 41)$. Cette prière, concluant le premier chapitre Die große Hoffnung, montre à l'évidence que l'écrivain ne se propose pas de rédiger un livre documentaire à caractère autobiographique, mais de sauver de l'oubli, surtout de transmettre à l'aube des temps nouveaux, par un récit poétique $^{7}$, l'appel des anges et l'éclat des poussières d'étoiles ayant guidé dans les ténèbres les pas hésitants de l'enfant. Elle a dû affronter la pire des situations qui soit, celle de la double non-appartenance: «Unentschieden, das ist das Ärgste» (gH, 44), imputable à l'existence de deux grands-parents « corrects » et de deux qui ne l'étaient pas.

6 Dans la prose poétique de jeunesse d'Ilse Aichinger qui s'épure au fil des années pour n'être plus que dire poétique enchâssant les intervalles du silence, les références bibliques abondent; aux yeux du lecteur attentif qui, à la suite d'Ellen se met en marche, moins pour sonder le mystère de l'existence humaine que pour tenter de capter le secret de l'écriture, elles forment la trame même du livre. La fugitive allusion au récit yahviste de la Création retraçant le drame originel et ses conséquences pour la destinée de l'homme ( $\mathrm{gH}, 151,318)$, la mention discrète du premier homicide de l'histoire ( $\mathrm{gH}, 70)$ et des larmes du Psaume versées par les femmes exilées à Babylone (gH, 274) confèrent à l'évocation de la souffrance humaine une dimension mythique. Celle-ci est renforcée par les accents apocalyptiques des chapitres constituant la seconde moitié du livre où, de façon paradoxale, sont décrites simultanément une sorte de descente aux enfers (arrestation des enfants juifs, suicide de l'aïeule, anéantissement de la ville sous le feu des bombardements) et la course folle de l'héroïne parmi les agents de la destruction dans les rangs desquels elle opère, grâce à son obstination et son impressionnante vitalité, des prodiges éphémères. Cette force indéfectible et fascinante, Ellen la doit à sa recherche du plus grand espoir, de la Terre Sainte dont la frontière est entrevue en état de demi-sommeil avec le groupe d'enfants juifs pendant une équipée nocturne fantastique dans un corbillard.

7 Trois énigmatiques personnages issus de l'histoire sacrée et profane se joignent à eux. Un rite de passage est accompli avec le concours de l'homme à la cornemuse, Saint Augustin, celui qui a franchi la mer pour convertir les Angles, avec l'aide d'un jeune berger muni d'une lyre et d'une fronde, le roi David, et enfin de Christoph Colomb, le navigateur; tous trois sont détenteurs d'un message porteur de liberté : le doute, la souffrance et la vigilance dessillent les yeux des hommes, ils permettent de franchir la frontière de la Terre Sainte qu'il convient de redécouvrir. 
Comme le constate Brita Steinwendtner avec perspicacité à propos d'une publication ultérieure d'Ilse Aichinger :

Nichts bleibt, wie es ist, nichts, was es ist, wenn Ilse Aichinger es anrührt: Zusammenhänge, Vorstellungen, Tatsachen, Wörter. Alles verliert seinen Boden, seine Bestimmung, sein Festgefügtes. Use Aichinger verändert, entgrenzt. Sie ist und macht widerständig8.

9 La remarque vaut également pour Noé, le vieil homme de la scène suivante qui, lors d'une leçon d'anglais sur une toile de fond métaphorique de déluge et de naufrage du sens, contribue lui aussi à assurer le passage, à faire entrevoir aux enfants cloués sur le sol natal le mystère du plus grand espoir :

Weshalb lernt man englisch? Um zu fliehen und nach einem Gasthaus zu fragen in dem fremden Land? Und das soll alles sein? Nein, das ist es nicht!... Jetzt fragt ihr andere Dinge! Der Zweck ist gefallen. Der Zweck ist nur ein Vörwand, nur ein Schatten des Wirklichen. Der Sinn bleibt. Wenn ihr jetzt noch englisch lernt, so lernt ihr es, ... um zu spielen vor dem Unbekannten! [...]

Alles, was wir tun, tun wir aus einem tiefen und geheimnisvollen Grund! [...]

Weshalb pfeifen die Drosseln? Weshalb reiten die Wolken? Weshalb funkeln die Sterne? (gH, 146-147)

10 Tous sont " au service d'une puissance étrangère », répond par anticipation le jeu de mots servant de titre à la séquence. Il n'est pas étonnant que dans cette découverte par à-coups des mystères de la destinée humaine l'ange, messager étrange sillonnant l'histoire biblique, prenne un relief particulier.

11 La figure de l'ange n'apparaît pas seulement dans le roman, elle ressurgit quelques années plus tard dans un récit intitulé Engel in der Nacht (1949)9. Elle y occupe une place centrale qui révèle son importance dans l'imaginaire de l'écrivain. En outre, elle est également mentionnée à deux reprises dans des textes autobiographiques assez brefs des années 60, évoquant les éprouvantes années de la jeunesse viennoise pendant la dictature nazie ${ }^{10}$. L'ange fait aussi l'objet de la première note du journal que l'écrivain rédige en 1951 :

Jahresanfang: Aus den Wurzelgruben der umgestürzten Eichen erheben sich die Erwachenden, die Engel, und verlassen die Gärten. Und übersteigen die Zäune mit geschlossenen Flügeln, an denen noch welkes Laub vom Vorjahr haftet [...] Und gehen Hand in Hand die Wege und nehmen die Erinnerung wie Schnee, der die Gruben füllt ${ }^{11}$.

12 Il faut retenir de cette réflexion aux tonalités énigmatiques ${ }^{12}$ les éléments qui accompagnent l'apparition : le réveil, les ailes et la neige.

S'il arrive dans la Bible que les anges soient représentés sous forme de jeunes hommes sans ailes, comme c'est aussi le cas jusqu'au IV siècle dans l'iconographie chrétienne, les ailes constituent dans la prose de jeunesse d'Ilse Aichinger l'attribut premier de ces messagers mystérieux. Seule exception : l'ange du jeu de la Nativité. Toutefois Léon, tantôt désigné par son prénom, tantôt par son rôle, et qui apparaît empêtré dans un vieux drap retenu par une épingle de sûreté rouillée, garde ostensiblement jusqu'à l'irruption de l'agent de la Gestapo qui va les livrer, un geste caractéristique de sa fonction : les bras grands ouverts. 

le titre du huitième chapitre (Die zerbrochenen Flügel) où s'affrontent les forces de la vie et celles de la mort dans un combat indécis qui s'achève comme un conte sur le chant d'un cortège d'enfants célébrant Saint Nicolas, celui qui peut substituer les ailes aux bottes.

\section{accompagnent l'irruption du
autobiographique postérieur}

Damals, als die äußere Bedrängnis der inneren zur Hilfe kam und beide zusammen

wie zwei Engel den Augenblick wieder in sein Recht setzten ${ }^{13}$.

Mais il ne s'agit pas exclusivement d'une souffrance liée aux persécutions raciales. Engel in der Nacht se déroule, quant à lui, pendant la période de reconstruction. Les protagonistes sont un couple d'enfants placés dans une situation de seuil portant le sceau de la complexité. La sœur, une adolescente, s'achemine vers l'âge adulte tandis que le cadet refuse de s'engager à sa suite, car l'enfant a l'intuition que ce passage le condamne à une plongée sans retour dans un réel ayant perdu toute profondeur.

18

Deux grandes parties composent le texte. Dans un premier temps le jeune garçon s'efforce en vain de percevoir les anges qui, à l'aube, selon la jeune fille, emplissent le ciel pour annoncer la Nativité prochaine. Sa crédulité lui vaut des moqueries à l'école jusqu'au jour où sa mère, vivant en dehors du foyer, répond par un rire à l'interrogation de l'enfant. Ce soir-là éclate la grande révolte du garçon contre le monde désormais dépourvu de sens et contre la sœur dont le mutisme et la passivité inexplicables le désarment. C'est alors que s'ouvre dans la nuit une lutte acharnée pour reconquérir les anges : ils finissent par apparaitre dans une vision fantastique, puis se dérobent à la vue et au toucher du chercheur obstiné qui s'empare du rôle de guetteur de l'aînée alors mystérieusement disparue et retrouvée morte aux premières lueurs du jour : elle gît en bas, dans la neige, sous la fenêtre où s'opéra la vision.

19 Tous les grands tableaux où les anges, voire - selon un procédé de métonymie - leur attribut, jouent un rôle de premier plan : Das große Spiel (gH, 188-234), Die zerbrochenen Flügel (gH, 276-317) et Engel in der Nacht (1949) sont toujours la description d'un combat dans lequel des enfants s'opposent, si ce n'est aux forces des ténèbres, du moins à celles des adultes glissant à la surface du réel, par passivité, confort ou désarroi ${ }^{14}$.

20 Le jeu de la Nativité qu'Ellen et ses amis désemparés s'acharnent à jouer dans la pénombre pour juguler la peur a été écrit par Anna, la veille du départ de son convoi pour la Pologne. La jeune fille dont le visage reflète la clarté de l'étoile leur a donné un conseil : «Geht dem Stern nach! Fragt nicht die Erwachsenen! Sie täuschen euch, wie Herodes die heiligen drei Könige täuschen wollte ! Fragt euch selbst, fragt eure Engel!» (gH, 185), puis elle leur a remis le texte avec ces paroles: «Da gibt es drei heilige Könige, einen Engel und noch alles mögliche! Ich glaube - es ist das Richtige!» (gH, 186). Il est significatif que les seuls personnages mentionnés soient le messager de la Nouvelle Alliance de Dieu avec les hommes et les vagabonds («Landstreicher»), épuisés et découragés par leur vaine recherche de la Paix à la suite de la mystérieuse étoile. «Spielt weiter», telle est la pressante et obsédante réplique de Léon, ange par 
procuration et metteur en scène par vocation, qui scande le jeu à chaque alerte de la sonnette lorsque l'effroi s'abat sur le groupe. La porte finit par s'ouvrir sur Ellen éplorée qui, ignorant le code, prend la mesure douloureuse de sa non-appartenance. Puis la lumière crue du vestibule signale la venue de la dame d'à côté; elle est caractérisée par sa valise, sa précipitation, son trouble face à la subversion tranquille et résolue des enfants qui refusent les limites glacées de la raison. Contrairement à l'adulte, ils savent - comme le leur a révélé le jeune roi David - où se trouve la Terre Sainte :

Überall dort, wo Menschen Schafe hüten und alles verlassen, wenn der Engel ruft $(\mathrm{gH}, \mathrm{III})^{15}$.

21 L'issue de la dernière confrontation avant l'arrivée de la Gestapo est plus indécise. L'ultime adversaire est le monsieur d'en face, le monsieur «bien informé » qui avec le geste de l'ange, bras tendus, proclame: «N'ayez pas peur, les déportations sont suspendues ». D'abord incrédules et troublés, les enfants finissent par se rallier à Ellen la rebelle, puis à Léon et ils résistent à la séduction, affrontent le faux prophète irrité et le contraignent à s'intégrer au jeu : il sera le quatrième vagabond-roi. C'est à Léon l'ange de la scène - que revient alors de prononcer la parole décisive :

Israël heißt Gottesstreiter ! Wir heißen alle Israël !

Anordnung der geheimen Polizei $(\mathrm{gH}, 228)^{16}$.

22 Dans le chapitre plus tardif Die zerbrochenen Flügel où est décrit un long interrogatoire dans un poste de garde, Ellen réussit un extraordinaire travail de sape auprès du colonel et de ses matiques à force d'ingénuité et de conformité à la logique intérieure de l'adolescente manifestement fondée sur le message de l'ange :

Warum habt ihr eure Flügel zerbrochen und gegen Stiefel vertauscht? Wer Stiefel trägt, kann kein Visum bekommen. Barfuß muß man über die Grenze gehen [...]. Der Himmel ist unterwegs, aber ihr haltet ihn auf (gH, 307).

23 Et face à ses hommes qui sont sur le point de « se réveiller ", le colonel doit exhorter les troupes à la vigilance : «Hört und seht, aber horcht nicht und schaut nicht, denn es führt zu weit...» $(\mathrm{gH}, 311)$.

Être un guetteur vigilant, c'est ce que devient, quant à lui, le narrateur du récit, à l'aube, après son combat acharné pour reconquérir les anges. La vision des créatures célestes s'achève sur un appel au réveil de tous les dormeurs ou de tous les automates aveugles, comme ce père, ombre éternellement absente qui, au lieu de veiller, sillonne vainement les rues de la cité à la recherche de cadeaux illusoires.

Ce n'est pas un hasard si tous ces combats ont lieu dans l'obscurité de l'automne finissant, moment de l'année où se succèdent la fête juive des Lumières et la célébration chrétienne de l'Avent. L'Avent qu'évoque si souvent l'écrivain n'est pas un rituel profane fait de guirlandes de pacotille, de cheveux d'ange de contrefaçon et de sapins trop tôt coupés pour parer la ville ou être vendus par des marchands assoupis ${ }^{17}$. Ilse Aichinger qui, comme Ellen croit au Dieu nouveau, chante l'attente de Noël «dieses lebengebenden Augenblicks» ${ }^{18}$, ce temps de veille nostalgique vécu par l'enfant et qui reste inséparable de l'image de l'ange :

... ein Teil des Raums, ein dunkler stiller Flügel, der sich gefaltet hatte über dem Rattern der Straßenbahnen, dem Küchenlärm am Sonntag, der Stimme des Geographielehrers am Vormittag. Etwas, das zugleich abdämpfte und deutlich machte $^{19}$. 
ces combats sont étroitement associés à un moment de la journée situé entre le crépuscule et l'aube. La présence du veilleur parmi les hommes endormis possède à l'évidence une signification métaphorique renvoyant à la situation de crise ou de désespoir. Sans doute peut-on voir aussi dans les indications horaires précises du récit (à la tombée de la nuit commence l'affrontement avec la sœur, à six heures le lendemain la vision s'achève) l'évocation du prodige que constitue toujours le début d'un jour nouveau. Suscité par l'invocation des anges, il correspond à la fois à l'aube et à la pratique juive de comptage des jours, d'un coucher de soleil à l'autre.

Anges et prodiges vont de pair dans la prose poétique de l'écrivain autrichien. Aussi n'est-il pas surprenant que la neige et sa blancheur éblouissante, agent de dissimulation et de métamorphose, accompagne le plus souvent l'émergence du thème de l'ange. C'est dans une tempête de neige que surgit dans le récit la vision de l'armée céleste, et c'était déjà l'intuition de la neige prochaine qui introduisait le chant des enfants célébrant Saint Nicolas, celui qui rend leurs ailes aux hommes capables de déposer sur le rebord de la fenêtre leurs bottes, symbole de l'assurance grégaire et de l'oppression.

Ouverture banale de constructions ordinaires, la fenêtre devient chez Ilse Aichinger le lieu où se manifeste - pour reprendre l'expression de M. Eliade ${ }^{20}$ - « une rupture temporaire dans l'homogénéité de l'espace » où le passage de la réalité objective au surnaturel est rendu possible, c'est-à-dire du monde profane au monde sacré.

Rien ne peut par exemple empêcher les bourrasques de la tempête de neige de s'y engouffrer et d'apporter la révélation à l'enfant. Ailleurs, c'est un désir irrésistible qui pousse les deux hommes du poste de garde chargés de la surveillance d'Ellen à se lever pour ouvrir les fenêtres et à se pencher à l'extérieur lorsque résonne la voix claire des enfants. En marche à la suite du saint évêque, ils manifestent à leur insu l'incroyable nouvelle : «Der Mann im Mond war zur Erde gestiegen » $(\mathrm{gH}, 316)$. Enfin, c'est perché sur la fenêtre occultée du logis clandestin que l'ange du jeu de la Nativité lance dans un soupir que le temps de Noël est arrivé (gH, 196).

a fenêtre devient - pourrait-on dire en référence au songe de Jacob en route pour Harân - la " Porte des Cieux ${ }^{21}$, « le point paradoxal de passage d'un mode d'être à un autre $^{22}$ ", le lieu éphémèrement sacralisé où est délivrée, par le truchement des anges, la révélation du mystère de l'amour divin.

Par son écriture Ilse Aichinger bouscule le sens convenu des mots, des choses et des images, le régénère pour réveiller les hommes "endormis » et restituer la vue aux «aveugles». Différentes questions se posent alors quant à la mise en œuvre du matériau biblique relatif aux anges. La première concerne l'ampleur de l'intervalle posé entre les Saintes Écritures et la fiction poétique, la seconde la teneur des acquis et la portée du message.

Comme le rappelle Pierre Cazier ${ }^{23}$, l'utilisation de la Bible dans la création littéraire doit être distinguée de celle de la mythologie en générale. À ceci, plusieurs raisons : malgré la diversité des auteurs, la Bible est d'abord considérée comme un livre unique inspiré par Dieu, ensuite elle se présente comme un texte véridique affirmant l'action de Dieu sur l'histoire et enfin, elle bénéficie d'un statut de référence culturelle commune au 
plus grand nombre à cause de la continuité de sa propagation au cours des célébrations religieuses. Il est permis d'ajouter que cette imprégnation culturelle est particulièrement marquée dans l'espace germanique.

À la suite des représentants les plus prestigieux des lettres allemandes, Ilse Aichinger subit, comme ses contemporains, l'influence des textes bibliques qui nourrissent sa foi et son imaginaire, façonnent sa pensée, imprègnent son écriture. En outre, sa situation particulière au carrefour des cultures juive et chrétienne à une époque rongée par un antisémitisme dévastateur est déterminante. Catholique, elle fréquente dès son jeune âge des écoles religieuses, adolescente, elle participe aux activités de la Hilfsstelle fur nicht arische Katholiken dans les locaux du palais archiépiscopal mis à leur disposition par le cardinal Innitzer ${ }^{24}$. Par ailleurs, elle voue depuis toujours un véritable culte à sa grand-mère maternelle et partage depuis 1938 le sort des persécutés du nationalsocialisme constituant - depuis la séparation des parents (1927) - la cellule familiale. La jeune fille est donc simultanément imprégnée de culture judaïque vivante et de foi chrétienne. Et dans les années noires, l'enjeu n'est pas pour elle tant de survivre que d'être mue par la vraie vie puisée à la source de l'Ineffable. Dans ce contexte, la mention fréquente des anges dans la prose de jeunesse et les écrits auto-biographiques postérieurs dépasse le cadre de la création littéraire, elle manifeste une indéniable dimension existentielle.

Le Dictionnaire de la Bible et des trois religions du Livre, édité par André Chouraqui ${ }^{25}$, propose une définition du mot "ange ", correspondant à la signification que lui prête Ilse Aichinger dans sa prose poétique: "Le mot ange dérive du latin angélus qui transcrit, au lieu de le traduire par nuntius, le grec aggelos. Ce dernier traduit exactement l'hébreu maVak, "messager", qui désigne une fonction et non une nature, comme en est venu à signifier le mot ange ». Ceci conduit tout naturellement à recenser les différentes fonctions de l'ange dans la Bible et à s'interroger sur les traces laissées dans les œuvres étudiées.

L'arrivée des anges dans l'Écriture correspond à l'évolution interne du judaïsme qui a progressivement épuré son monothéisme de ses aspects les plus anthropomorphiques. $\mathrm{Au}$ Dieu du récit yahviste rencontrant ses créatures pour converser avec elles se substitue un Dieu transcendant que l'on ne peut voir sans mourir : il envoie alors ses anges ou s'adresse aux hommes par des songes. L'apparition de l'ange est donc l'expression de la distance grandissante entre le Créateur et les hommes.

Des premières évocations de ces esprits célestes dans la Bible on ne relève que des traces diffuses chez Ilse Aichinger. On notera cependant l'ange de Yahvé, mentionné dans les couches les plus anciennes de l'Ancien Testament en tant que manifestation visible de Yahvé. Il se confond fréquemment avec lui (Gn 16,11-13) et serait, selon A. Chouraqui, un artifice littéraire pour voiler l'anthropomorphisme des anciens textes et préserver le secret de la grandeur de Dieu. De cet ange, il subsiste dans le roman une représentation atténuée dans l'évocation voilée d'un Dieu lointain mais aimant, au moment où les enfants partent à la recherche de la Terre Sainte en invoquant les ancêtres dans le vieux cimetière juif :

... In diesen Himmel, der wie ein Gesicht über ihnen war, wie das Erbarmen eines Fremden, wie Licht, das sich fallend verbirgt. In diesen Himmel, der schwerer und immer schwerer über sie sank, wie Flügel, die zu groß sind (gH, 76).

De la période préexilique en général où on ne discerne pas d'angélologie véritablement élaborée, les anges y apparaissent essentiellement comme les membres de la cour de 
Yahvé sans tâches précisément définies, on ne trouve chez Ilse Aichinger qu'un lointain écho. Il est perceptible d'une part, dans les armées d'anges vaincus qu'imagine le garçon du récit au moment où la sœur aînée se dérobe à l'affrontement ardemment désiré et d'autre part, dans la multitude des anges dont l'enfant soupçonne la présence derrière « son ange " dans les bourrasques de neige nocturnes.

Il faut attendre le temps des prophètes quand, outre la fonction de louange et d'exécution des sentences divines, apparaît le rôle d'interprète de la Parole de Dieu dévolu à certains anges, pour que des éléments significatifs de convergence entre l'Écriture et l'œuvre de Ilse Aichinger puissent être relevés. Encore faut-il préciser qu'il est ici question des prophètes de la période exilique et postexilique, de ces prophètes semeurs d'espérance qui sont les principaux organes du progrès de la révélation. Ils apportent aux hommes la consolation et la bonne nouvelle : le châtiment n'est pas le dernier mot de Dieu.

Les filiations les plus directes qui peuvent être établies entre la Bible et les textes étudiés, renvoient ainsi à des évocations d'anges indissociables d'hommes représentatifs de l'attente messianique et ayant eu une expérience immédiate de Dieu. Deux noms doivent ici retenir l'attention : ceux d'Ezechiel et de Daniel.

Dans la vision du Temple futur que reçoit Ezechiel intervient un ange désigné sous le terme d'« homme » (Ez 40,3). Il remplit le rôle d'interprète auprès de ce prophète dont le messianisme se pare de tonalités nouvelles : un nouveau David est annoncé, et il sera le « berger » de son peuple. Ainsi peut-on considérer que la figure du berger qui surgit parfois soudainement et mystérieusement dans les premières œuvres (gH, 354-357; $\mathrm{ENa}, 38)$ ne prend pas uniquement ses racines dans l'Evangile de Luc, mais bel et bien chez ce prophète qui partage avec le jeune écrivain un pouvoir d'imagination exceptionnel.

41 Mais selon toute vraisemblance c'est dans le Livre de Daniel que se trouve la source d'inspiration la plus plausible, de par les circonstances de sa rédaction, son style et la place prise par les anges. Sans doute composé entre 167 et 164 avant notre ère, il est destiné à soutenir la foi et l'espérance des Juifs persécutés par Antiochus Epiphane. Il annonce l'avènement d'un royaume gouverné par un fils d'homme et apporte, sur un mode volontairement énigmatique, la révélation du dessein de Dieu sur son peuple et sur tous les peuples, dévoilé par l'intermédiaire d'êtres mystérieux, qui sont les messagers du Très-Haut. Si l'on se souvient que l'ange Gabriel, envoyé dans un premier temps par Dieu pour «donner l'intelligence» d'une vision (Dn 8,16), fond ensuite en plein vol sur le prophète (Dn 9,21) qui est le seul à déceler sa présence, l'on comprend mieux certaines réflexions paradoxales du narrateur de Engel in der Nacht manifestant les voies impénétrables du Seigneur :

Damals wußte ich noch nicht, daß es die Engel sind, die uns beschwören. Nicht wir sind es, die sie erträumen, die Engel erträumen uns. Wir sind die Geister in ihren hellen Nächten... Wenn die Schatten über die Wüste fallen, wirft sie der Himmel (ENa, 36).

Enfin, on peut admettre que le motif du veilleur qui sillonne tout le récit repose sur l'allusion faite aux Vigilants (=Wächter) (Dn 4,10) dans le songe de Nabuchodonosor, ces Vigilants - terme propre à Daniel - qui deviendront les anges gardiens d'une tradition postérieure. Mais chez I. Aichinger il s'agit moins de protéger que de doter l'homme en recherche de la capacité de recevoir le message de l'amour divin. 
La doctrine des anges qui s'affirme chez ce prophète était déjà perceptible chez Ezechiel et dans le livre deutérocanonique de Tobie qui mérite lui aussi d'être mentionné. Certains traits caractéristiques de la figure de l'ange dans cette histoire sont repris par Ilse Aichinger. Raphaël, le guide de Tobie et l'artisan de la délivrance de Tobit et de Sarra, se présente à la fin du récit d'abord comme un intercesseur des hommes désespérés auprès de Dieu, puis surtout comme un envoyé du Seigneur chargé d'éprouver la foi et d'apporter la guérison ( $\mathrm{Tb} 12,12-14)$. Il manifeste ainsi la proximité d'un Dieu bienveillant, tout en restant lui-même une créature mystérieuse dont les hommes ne perçoivent que l'apparence (Tb 12,19). Aussi peut-on voir dans l'attitude distante de l'ange du récit le reflet des propos tenus par l'Archange à Tobie et à son père: en effet, bien que reconnu comme l'ange du narrateur, il répugne à laisser toucher l'ourlet de son vêtement malgré le désir brûlant de l'enfant ${ }^{26}$.

Il ressort de ces remarques que la fonction de l'ange, intermédiaire mystérieux entre le secret de l'amour divin et la souffrance des hommes, prend dans l'œuvre de jeunesse, une place centrale conforme au modèle biblique. Mais ce constat ne saurait entièrement rendre compte d'une caractéristique originale de la figure de l'ange manifestée nettement dans la mise en scène profane du jeu de la Nativité, à savoir la complexité de la relation décrite entre l'homme et l'ange. L'analyse du double personnage de Léon, l'ange d'un soir, peut à cet égard servir de révélateur.

Même si la désignation "ange " s'avère prépondérante dans Das große Spiel (36 occurrences contre 27 citations du prénom), il n'en est pas pour autant moins intéressant d'observer que la présentation de l'ange s'ouvre sur la peinture de la vulnérabilité humaine : il est un être désemparé, à l'aspect misérable. Son désarroi et ses hésitations, tantôt imputables au péril extérieur, tantôt aux commentaires désabusés de l'un des enfants, sont manifestes mais pourtant il est toujours celui qui réussit à se ressaisir. Il parvient à exhorter chacun à reprendre son rôle, afin de lutter contre l'emprise d'un découragement dont le lecteur ne sait plus exactement s'il résulte de la marche harassante des vagabonds ou s'il est suscité par l'angoisse qui tenaille les enfants. Non seulement «l'ange », mais aussi «Léon» sont une instance de décision pour le groupe. Léon est l'aîné, il les a tous initiés à la nage et au sauvetage et il est le type même du personnage qui chemine, mais sans précipitation. Des premiers tableaux ressortent son appartenance à la foi en "l'ancien Dieu ", sa plainte nostalgique devant les persécutions et ses interrogations solitaires. Mais progressivement se dessine une image nouvelle dont les caractéristiques essentielles sont un refus inébranlable de considérer l'étoile comme un insigne de mort et la volonté affirmée de suivre la voie ouverte par la clarté de l'étoile comme l'a enseigné Anna.

Lorsque «l'ange » se prononce finalement pour l'accueil du visiteur inconnu, il délivre un message dont la portée dépasse le cadre de la pièce de la Nativité :

Öffnet, öffnet jedem, der euch verlangt! Wer nicht öffnet, versäumt sich selbst (gH, 198).

Son enjeu ne se rapporte pas uniquement à la situation des enfants, mais suggère à l'homme la voie permettant d'accéder à la révélation divine.

Toutefois c'est «Léon », après un bref instant d'égarement au cours duquel il jette sur la petite troupe courageuse le regard à la fois ému de l'adolescent et distancié du metteur en scène qui, répondant à la prière d'une voix enfantine lui enjoignant de rester un ange, prononce le message d'espoir annonçant le mystère de l'amour et de la grâce divine : 


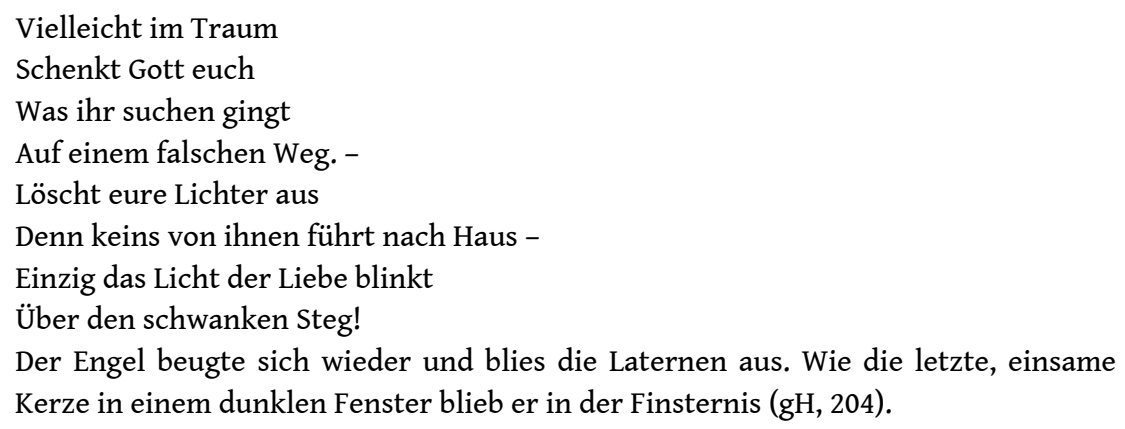

Le commentaire imagé et lapidaire, complétant ce qui peut être lu comme une didascalie, définit une conception de l'ange propre à Ilse Aichinger dans l'œuvre de jeunesse : il est le dernier recours, l'ultime rai de lumière dans les ténèbres, le don de Dieu aux guetteurs solitaires.

Quant au dernier message à l'énoncé paradoxal : «Denn man behält nur das, was man hergibt! » (gH, 211), il n'est plus ni le fait de «Léon », ni de «l'ange » de la tradition, mais d'une voix dont l'origine restera à tout jamais une énigme : «Niemals wurde es klar, wer dieses Wort gesagt hatte... Die leichte Stimme des Engels in einem finsteren Traum! Eine Stimme voll Erbarmen, die doch erbarmungslos die letzte Konsequenz verlangte» ( $\mathrm{gH}, 211)$. Un rapprochement de cette réflexion avec le conseil d'Anna: «Fragt euch selbst, fragt eure Engel» ( $\mathrm{gH}, 185)$ suggère que l'ange, messager de la révélation, de la miséricorde infinie, serait alors cette voix exigeante que l'homme, s'il est de la lignée des veilleurs, peut entendre dans le silence intérieur qu'il a su préserver («Stille setzte ein: das Stichwort für die Engel» gH, 202) et qui lui permet d'accéder au secret de la Création.

51 La révélation est un don de Dieu comme l'a proclamé l'ange, mais pour que sa parole n'erre pas dans la galaxie comme l'enfant Jésus qu'aucun messager ne vient plus révéler (ENa, 37,38), il faut que l'homme se donne les moyens de la recevoir, c'est-à-dire éveille l'ange qui est en lui. Pour ce faire il doit secouer tout ce qui le fige dans une relation stéréotypée au Créateur et le dépossède d'une sorte de sixième sens destiné à percevoir le réel au-delà de son écorce visible. C'est la teneur des propos chiffrés du narrateur dans le récit de 1949 : l'ange naît avec l'enfant, mais l'angelot joufflu à la chevelure bouclée se mue imperceptiblement en une créature aux ailes pesantes dont il faut se libérer, avant que le désir ardent de la quête de l'Ineffable ne s'éteigne irrémédiablement.

À propos du roman où se succèdent des miracles qui ne sauvent pas le corps mais l'âme, Erich Fried écrit de son exil londonien en 1949 :

Die größere Hoffnung ist ein tiefreligiöses christliches Buch. Auch in der Sprache, in den Paradoxien und tiefernsten Wortspielen, in den wundersamen mühelosen Übergängen aus dem Diesseitigen ins Jenseitige offenbart sich christliches Empfinden ${ }^{27}$.

On ne peut que souscrire à ce jugement : l'empreinte du christianisme est incontestable dans ce livre qui peut être interprété comme une affirmation graduelle de l'espérance apportée par l'incarnation du fils de Dieu. Elle se manifeste d'abord dans la mise en scène de personnages en situation de seuil : par exemple Ruth, fraîchement baptisée, elle chante avec les enfants et leur apprend - symboliquement - la troisième voix, ou bien encore la grand-mère, agitée dans son agonie. Après avoir en vain tenté de l'apaiser par la lecture de prières ancestrales, Ellen l'ondoie avec l'eau du verre ayant servi à l'absorption des dragées de poison, tandis que la croix du chapelet oscille et 
brille au-dessus de la portion de carte géographique où figure le Cap de Bonne Espérance...

C'est surtout dans la seconde partie de l'œuvre, lorsque se déchaînent les bombardements, que les allusions à la Nouvelle Alliance se font plus pressantes. L'ensevelissement temporaire d'Ellen et de deux cambrioleurs dans une cave est peutêtre une évocation symbolique de la Résurrection. Par ses murmures angoissés, Ellen initie l'un des deux larrons à croire aux anges qui ont poussé la pierre du tombeau ( $\mathrm{gH}$, 339). Lorsque sauvés, ils s'engagent dans une course folle à travers le brasier, titanesque "hémorragie du monde ", ils sont frappés par une soif torturante qui fait surgir sur un fond d'apocalypse les images enchevêtrées des noces de Cana et de la transsubstantiation. S'y mêlent aussi une évocation acoustique du flux de la Mer Rouge et du chant d'un jeune et mystérieux berger des temps anciens qui, aux portes d'un abattoir, exhorte les pillards à se dessaisir des biens illusoires pour mieux recevoir les dons de la vraie vie. Si «le plus grand espoir » peut être conçu comme un écho de la révélation de la Nouvelle Alliance conclue par Dieu avec tous les hommes appelés à être des guetteurs et des êtres de lumière, il exprime à l'évidence simultanément une foi ardente dans le Dieu d'Israël.

L'inspiration religieuse, résultat d'une expérience vivante des cultures judaïque et chrétienne et d'une situation paradoxale d'appartenance et de non-appartenance, confère au premier roman d'Ilse Aichinger une tonalité poétique particulière. Elle a permis en outre une mise en perspective historique précoce de la Shoah qui a valu à son auteur, lors de la parution du livre en Allemagne, un accueil réservé28. L'invocation des anges fait, quant à elle, ressortir la dimension existentielle de la mise en œuvre symbolique du modèle biblique dans la prose des années quarante. Cette écriture se propose moins de transmettre un message chrétien que d'exprimer l'univers intérieur de l'écrivain. Elle donne forme aux émergences du sacré, non dans le cadre d'une expérience conforme à une orthodoxie religieuse, mais à la lumière de la détresse des hommes de ce temps, au-delà même de la limite convenue de 1945.

Les nombreuses occurrences du motif des «ailes » attestent tantôt la possibilité d'un élan vers l'Ineffable, tantôt les pesanteurs de l'existence terrestre, et traduisent l'incompréhension devant l'énigme du destin de l'homme qui, créé pour être le reflet de l'amour divin, se charge de culpabilité et devient l'agent de son propre anéantissement, de son errance dans un univers de souffrance. Mais «l'appel» de l'ange prend aussi une autre signification, primordiale à ce stade de la création littéraire: il manifeste la foi dans la capacité indestructible de l'homme à être le premier artisan de sa rédemption, à se montrer un être pugnace décidé à arracher à Dieu un geste de miséricorde ${ }^{29}$, car il est habité par la nostalgie infinie de la réalisation de la promesse faite par l'ange de l'Apocalypse, celle d'un ciel nouveau et d'une terre nouvelle dont seront bannis la malédiction, la nuit, les cris et les larmes (Ap 21, 1-4 et Ap 22, 3-5). 


\section{NOTES}

1. - Ilse Aichinger : Die größere Hoffnung, Bermann-Fischer, Amsterdam 1948. Cette édition sert de référence (abréviation : gH).

2. - I. Aichinger : Rede unter dem Galgen, Jungbrunnen Verlag, Wien 1952. Le volume comprend les récits suivants : Das Plakat (1948), Spiegelgeschichte (1948-49), Rede unter dem Galgen (1949), Engel in der Nacht (1949), Mondgeschichte (1949), Der Hauslehrer (1949), Die geöffnete Order (1949).

3. - I. Aichinger : Der Gefesselte, Erzählungen (1948-52), Fischer Taschenbuch Verlag, Frankfurt a.M. 1991, p. 10.

4. - John A.S. Grenville : Die «Endlösung» und die «Judenmischlinge» im Dritten Reich, in: Das Unrechtsregime. Internationale Forschung über den Nationalsozialismus. Bd.2, hrsg. von U. Büttner, Hans Christians Verlag, Hamburg 1986, p. 91-121.

5. - John A.S. Grenville, op. cit., p. 91.

6. - Ces textes doivent être considérés comme des phases initiales de la version définitive des chapitres du roman : Das vierte Tor, in: Wiener Kurier, 1.9.1945 (cf. ch. 3, Das heilige Land). Der Kai, in: Der Turm, H. 5/6, 1947, p. 210- 212.

7. - Carine Kleiber: I. Aichinger, Lehen und Werk, Peter Lang, Bern 1984, propose cette dénomination empruntée à Jean-Yves Tadié : Le récit poétique, Presses universitaires de France, 1978.

8. - Brita Steinwendtner: Sammle den Untergang. Zu Ilse Aichingers Kurzprosaband «Schlechte Wörter» (1976), in: K. Bartsch und G. Melzer (Hrsg.): Ilse Aichinger, Literaturverlag Droschl, Graz 1993, p. 138.

9. - Edition de référence: I. Aichinger: Nachricht vom Tag, Erzählungen, Fischer Bücherei, Frankfurt a.M. 1970, p. 34-40 (abréviation : ENa).

10. - Vor der langen Zeit (1964), et Der erste September 1939 (1969), in: Kleist, Moos, Fasane, Fischer Taschenbuch Verlag, Frankfurt a.M. 1991, p. 19-22 et 23-27 (abréviation : K1MF).

11. - K1MF, op. cit., p. 49.

12. - La référence au dernier verset de la vision d'Isaïe $(6,13)$ : « La souche est une semence sainte " éclaire le sens de cette note.

13. - K1MF, op. cit., p. 21.

14. - Pour une interprétation détaillée se reporter à : B. Desbrière-Nicolas : «Wenn ihr nicht werdet wie die Kinder...» Ilse Aichingers Erzählungen in den 40er Jahren, in : Österreichische Literatur nach 1945. Die 40er und die 50er Jahre, Beiträge zur Robert-Musil-Forschung und zur neueren Österreichischen Literatur, Röhrig-Verlag, St. Ingbert, 1999 (Actes du colloque : Österreichische Literatur nach 1945, Université de la Sarre, 4-6 décembre 1997).

15. - La mention des bergers n'est pas uniquement la reprise de l'Evangile de Luc (2, 15-16) mais aussi une image de contraste servant de pendant à la condamnation par le Seigneur des mauvais bergers d'Israël (Ezechiel 34, 2, 10).

16. - Allusion au passage de la Genèse dans lequel Jacob après un corps à corps avec Dieu reçoit le nom d'Israël (Gn 32, 25-29) : «On ne t'appellera plus Jacob, mais Israël, car tu as été fort contre Dieu, et contre les hommes tu l'emporteras ».

17. - Ces motifs fugitivement insérés dans le récit $(\mathrm{ENa}, 34,35,37)$ réapparaissent dans le texte autobiographique Vor der langen Zeit (1964).

18. - K1MF, op. cit., p. 21.

19. - Ibid. p. 20.

20. - Mircea Eliade : Le sacré et le profane, Gallimard, col. Folio Essais 82, Paris 1997, p. 26.

21. - Gn28, 12-17.

22. - M. Eliade, op. cit., p. 30. 
23. - Pierre Cazier : La Bible et la création littéraire : de la théorie patristique à la pratique profane, in : Uranie, Mythes et Littératures, 1/1991, p. 53-70.

24. - Voir à ce propos :

I. Aichinger : Hilfsstelle, in: K1MF, Op. cit., p. 28-31.

Richard Reichensperger: Orte. Zur Biographie einer Familie, in: K. Bartsch und G. Melzer (Hrsg.), op.cit., p. 231-247. L'éditeur des œuvres complètes d'Usé Aichinger y fait état de l'origine autobiographique des chapitres Das große Spiel et Im Dienst einer fremden Macht.

25. - André Chouraqui : L'univers de la Bible, t. X, édition Lidis, 1985.

26. - Anne-Marie Bouisson interprète cette distance insurmontable entre l'ange et l'enfant comme le signe d'une évolution de l'écrivain. L'ange n'apporte plus la certitude aux cœurs inquiets (cf. Das große Spiel), « Il devient symbole de l'incertitude acceptée inhérente à l'humaine condition. » In: Austriaca, n 7, 1978, p. 17, Ilse Aichinger : approche d'une évolution.

27. - Erich Fried: Die größere Hoffnung, 1949, in: S. Moser (Hrsg.), Ilse Aichinger, Leben und Werk, Fischer Taschenbuch Verlag, Frankfurt a. M. 1995, p. 155-156.

28. - Heidi Kaiser analyse, dans le cadre d'une réflexion sur le dire poétique après Auschwitz, les reproches majeurs formulés par la critique allemande, in: H. Kaiser, G. Köpf (Hrsg.) : Erzählen. Erinnern., Verlag Moritz Diesterweg, Frankfurt a. M. 1992, p. 20-21. Ces reproches sont de deux ordres: d'abord la poétisation du monde qui banaliserait les horreurs commises, et ensuite l'évocation de la situation d'Ellen dans une perspective existentielle plus large qui conduirait à une généralisation inacceptable, tendant à relativiser la Shoah.

29. - Jacob qui, après un combat acharné avec Dieu lui arrache sa bénédiction et reçoit le nom d'Israël, est sans doute le patriarche tutélaire célébré ici (Gn 32, 25-30).

\section{RÉSUMÉS}

Ilse Aichinger note en 1951 dans son journal : « ... le ciel ne s'ouvre plus nulle part». Cette réflexion quelque peu énigmatique et empreinte de pessimisme ne manque pas d'étonner le lecteur de l'œuvre de jeunesse pour lequel la référence à la Bible traduit les mystères de l'amour du Dieu créateur. Dans le premier roman Die größere Hoffnung (1948), il s'agit en effet pour la jeune femme qui, en raison de sa condition de «demi-juive» a partagé l'angoisse des enfants juifs, de suggérer par l'intermédiaire des prophètes et des anges les possibilités de la rédemption par la souffrance. Le récit de 1949 intitulé Engel in der Nacht montre, quant à lui, par le songe d'un garçon confronté à la situation critique du passage à l'adolescence, puis à l'âge adulte, la nécessité pour l'homme de rester, sa vie durant, un veilleur. L'étude de l'ange dans l'œuvre de jeunesse permet de mieux appréhender les facteurs déclencheurs d'écriture chez le jeune écrivain au style si particulier. L'article se propose d'abord d'examiner les circonstances conduisant à l'émergence du personnage de l'ange, puis de mettre au jour les relations qui existent entre le modèle biblique et sa mise en œuvre littéraire. Enfin, il tente de cerner l'ambivalence caractérisant l'appel de l'ange, à la fois messager de l'Ineffable et voix de l'homme abandonné. Il apparaît ainsi que l'invocation des anges dans les deux textes analysés manifeste avant tout le brûlant désir de surmonter la coupure entre le Créateur, ressenti comme inaccessible, et l'humanité souffrante.

«... der Himmel ist nirgends mehr offen» trägt die dreißigjährige Ilse Aichinger 1951 in ihr Tagebuch ein. Jedoch dienten einige Jahre zuvor die ins Werk eingeführten Zitate aus den 
Prophetenbüchern nicht dazu, eine resignierende Weltanschauung zum Ausdruck zu bringen, sondern das Geheimnis von der Liebe Gottes zu verkünden. Damals half der Glaube an die Engel dem Menschen aus der Not: sowohl der Dichterin selbst, deren Erlebnisse in der jüngsten Vergangenheit als «Mischling ersten Grades» sich kaum von denen der jüdischen Kinder in ihrem ersten Roman Die größere Hoffnung (1948) unterschieden, als auch dem Jungen in der Erzählung Engel in der Nacht (1949), der auf der Schwelle zum Erwachsenenalter sich in den Nachkriegsjahren vom blendenden Licht des Scheinlebens bedroht fühlte. Die Figur des Engels in den vierziger Jahren ist zwar nicht vorherrschend, aber sie liefert immerhin Aufschlüße über Ilse Aichingers Schreibanlaß und deren eigenartige Schreibweise. Der vorliegende Aufsatz geht zuerst den Umständen der Engelerscheinungen nach. Es wird weiterhin versucht, die Abweichungen von der jüdischhristlichen Vorlage festzustellen und den ambivalenten Ruf des Engels als Boten des Unfaßbaren und als Stimme des ausgesetzten, rebellierenden Menschen zu untersuchen. Daraus geht deutlich hervor, daß die Beschwörung der Engel im Erstling und in der Erzählung die Sehnsucht nach Bewältigung der zwischen dem Schöpfer und seinen leidenden Kreaturen eingetretenen Entfremdung widerspiegelt.

\section{AUTEUR}

\section{BRIGITTE DESBRIÈRE-NICOLAS}

Université Charles-de-Gaulle - Lille III 